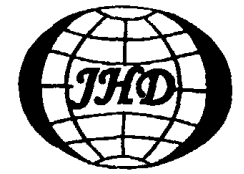

www.sciencedirect.com/ science/journal/10016058

\title{
THE PRESSURE TRANSIENT ANALYSIS OF DEFORMATION OF FRACTAL MEDIUM*
}

\author{
ZHANG Yi-gen \\ Institute of Mechanics, Chinese Academy of Sciences, Beijing 100080, China \\ Research Institute of Geological Sciences, Shengli Oilfield Company, Sinopec, Dongying 257015, China \\ TONG Deng-ke \\ School of Mathematics and Computational Sciences, China University of Petroleum (East China), Dongying \\ 257061, China, E-mail : tongdk@mail.hdpu.edu.cn
}

(Received February 13, 2007, Revised June 15, 2007)

\begin{abstract}
The assumption of constant rock properties in pressure-transient analysis of stress-sensitive reservoirs can cause significant errors in the estimation of temporal and spatial variation of pressure. In this article, the pressure transient response of the fractal medium in stress-sensitive reservoirs was studied by using the self-similarity solution method and the regular perturbation method. The dependence of permeability on pore pressure makes the flow equation strongly nonlinear. The nonlinearities associated with the governing equation become weaker by using the logarithm transformation. The perturbation solutions for a constant pressure production and a constant rate production of a linear-source well were obtained by using the self-similarity solution method and the regular perturbation method in an infinitely large system, and inquire into the changing rule of pressure when the fractal and deformation parameters change. The plots of typical pressure curves were given in a few cases, and the results can be applied to well test analysis.
\end{abstract}

Key words: stress-sensitive reservoir, fractal, permeability modulus, pressure analysis

\section{Introduction}

Numerous experiments have proved that the formation of oil reservoir and the fracture network distribution of fractured reservoir are fractal structures. Therefore, the flow theory of fractal reservoir has been developed and applied to oilfield. Fluid flow in hydrocarbon reservoirs and ground water aquifers have been traditionally studied by assuming the formation permeability is constant $t^{[1-6]}$. These assumptions in the fluid flow analysis have given good results in many situations, but with increasing exploitation of petroleum and geothermal resources from low-permeability and fractured formations, these

* Project supported by the National High Technology Research and Development Program of China (863 Program, Grant No. 2006AA06Z236), the Natural Science Foundation of Shandong Province (Grant No. Y2007F 13).

Boigraphy: ZHANG Yi-gen (1965-), Male, Ph. D. Student, Senior Engineering assumptions need to be re-evaluated. Kikani ${ }^{[7]}$ presented the flow model for cylindrical flow systems of deformed media. A perturbation technique was applied to determine the approximate solution and analyze the flow characteristics of deformed media reservoir. Yeung ${ }^{[8]}$ considered the spherical flow problem of deformed media reservoir. A simple technique was applied to obtain approximate solutions, but the error is large. The generalized pseudo pressure function was introduced to characterize the gas flow in pressure-sensitive reservoir ${ }^{[9]}$. The flow analysis for stress-sensitive reservoirs with double porosity was studied ${ }^{[10-16]}$. But the flow analysis for stress-sensitive fractal reservoirs has not been performed. Radial and spherical flow in homogeneous reservoir are special cases of fractal reservoir ${ }^{[1]} \quad\left(d_{f}=2\right.$ or 3 , and $\theta=0$ ). In this article, the fractal and deformed characteristics of stress-sensitive reservoir are considered. A permeability modulus is introduced to 
derive the radial flow equation for the stress-sensitive fractal reservoir. The perturbation solutions for a constant pressure production and a constant rate production of linear-source well are obtained by using the self-similarity solution method and the regular perturbation method in an infinitely large system, and inquire into the changing rule of pressure when the fractal and deformation parameters change.

\section{Flow equation}

The following assumptions are made in constructing the mathematical model:

(1)The permeability is stress-sensitive, that is, it depends on pore pressure.

(2)The porous medium is the fractal system with similar structure, the fractal permeable network embedded in impermeable Euclidean matrix, where the fractal network dimension is $d_{f}$, and the Euclidean matrix dimension is $(d=1,2,3)$.

The permeability modulus is defined as ${ }^{[1]}$

$$
\gamma=\frac{1}{k} \frac{\mathrm{d} k}{\mathrm{~d} p}
$$

The parameter $\gamma$ plays a very important role in the system where changes in effective stress affect the permeability. Basically, it measures the dependence of formation permeability on pore pressure. For practical purpose, $\gamma$ can be assumed as a constant.

Thus the permeability of fractal reservoir varies exponentially with pore pressure

$$
k=k_{0} \mathrm{e}^{-\gamma\left(p_{0}-p\right)}\left(\frac{r}{r_{w}}\right)^{d_{f}-\theta-d}
$$

where $k_{0}, p_{0}$ are initial permeability and initial pressure respectively, $r, r_{w}$ are the radial distance from well and the radius of wellbore respectively, and $\theta$ is the fractal diffusion exponent.

The continuity equation for the flow of a single-phase liquid in an isotropic and fractal reservoir can be given by assuming fluid to be slightly compressible and using Darcy's law, which is

$$
\frac{1}{r} \frac{\partial}{\partial r}\left(r \rho \frac{k}{\mu} \frac{\partial p}{\partial r}\right)=\frac{\partial(\phi \rho)}{\partial t}
$$

where $\rho=\rho(p)$ is density, and $\mu$ is viscosity.

Expanding Eq.(3) under the assumption of pressure-dependent rock properties leads to

$$
\begin{aligned}
& \frac{\partial^{2} p}{\partial r^{2}}+\frac{\beta}{r} \frac{\partial p}{\partial r}+\left(\gamma+c_{L}\right)\left(\frac{\partial p}{\partial r}\right)^{2}= \\
& \frac{\phi_{0} \mu}{k_{0}}\left(\frac{r}{r_{w}}\right)^{\theta}\left(c_{L}+c_{m a}\right) \mathrm{e}^{-\left(c_{m a}-\gamma\right)\left(p_{0}-p\right)} \frac{\partial p}{\partial t}
\end{aligned}
$$

where

$$
\begin{aligned}
& c_{L}=\frac{1}{\rho} \frac{\partial \rho}{\partial p}, c_{m a}=\frac{1}{\phi} \frac{\partial \phi}{\partial p}, \\
& \phi=\phi_{0} \mathrm{e}^{-c_{m a}\left(p_{0}-p\right)}\left(\frac{r}{r_{w}}\right)^{d_{f}-d}
\end{aligned}
$$

Then, assume $\gamma \gg c_{L}$, then Eq.(4) becomes

$$
\begin{gathered}
\frac{\partial^{2} p}{\partial r^{2}}+\frac{\beta}{r} \frac{\partial p}{\partial r}+\gamma\left(\frac{\partial p}{\partial r}\right)^{2}= \\
\frac{\phi_{0} c_{t} \mu}{k_{0}}\left(\frac{r}{r_{w}}\right)^{\theta} \mathrm{e}^{\gamma\left(p_{0}-p\right)} \frac{\partial p}{\partial t}
\end{gathered}
$$

where

$$
c_{t}=c_{L}+c_{m a}
$$

For the case of production of fluid at a constant rate from an infinite reservoir into wellbore, the dimensionless groups are defined as

$$
\begin{aligned}
& p_{D}=\frac{2 \pi k_{0} h\left(p_{0}-p\right)}{\mu q}, t_{D}=\frac{k_{0} t}{\phi_{0} \mu c_{t} r_{w}{ }^{2}}, \\
& r_{D}=\frac{r}{r_{w}}, \alpha_{D}=\frac{\mu q \gamma}{2 \pi k_{0} h}
\end{aligned}
$$

Under the constant-pressure production condition, the dimensionless groups are defined as

$$
p_{D}=\frac{p_{0}-p}{p_{0}-p_{w}}, t_{D}=\frac{k_{0} t}{\phi_{0} \mu c_{t} r_{w}^{2}},
$$

$r_{D}=\frac{r}{r_{w}}, \alpha_{D}=\gamma\left(p_{0}-p_{w}\right)$ 
In dimensionless coordinates, Eq.(5) becomes

$\frac{\partial^{2} p_{D}}{\partial r_{D}{ }^{2}}+\frac{\beta}{r_{D}} \frac{\partial p_{D}}{\partial r_{D}}-\alpha_{D}\left(\frac{\partial p_{D}}{\partial r_{D}}\right)^{2}=r_{D}^{\theta} \mathrm{e}^{\alpha_{D} p_{D}} \frac{\partial p_{D}}{\partial t_{D}}$

\section{The line-source solution for deformed fractal} reservoir

The mathematical model is made of Eq.(6) and initial and boundary value conditions.

(1)The flow problem of fluid in an infinite reservoir with the constant rate production is as follows:

$$
\frac{\partial^{2} p_{D}}{\partial r_{D}^{2}}+\frac{\beta}{r_{D}} \frac{\partial p_{D}}{\partial r_{D}}-\alpha_{D}\left(\frac{\partial p_{D}}{\partial r_{D}}\right)^{2}=r_{D}{ }^{\theta} \mathrm{e}^{\alpha_{D} p_{D}} \frac{\partial p_{D}}{\partial t_{D}}
$$

$p_{D}\left(r_{D}, 0\right)=0$

$$
\lim _{r_{D} \rightarrow 0}\left(r_{D}^{\beta} \mathrm{e}^{-\alpha_{D} p_{D}} \frac{\partial p_{D}}{\partial r_{D}}\right)=-1
$$

$\lim _{r_{D \rightarrow \infty}} p_{D}=0$

Rewrite the unknown function as

$p_{D}\left(r_{D}, t_{D}\right)=-\frac{1}{\alpha_{D}} \ln \left[1-\alpha_{D} \eta\left(r_{D}, t_{D}\right)\right]$

Equations (7)-(10) can be simplified as

$\frac{\partial^{2} \eta}{\partial r_{D}^{2}}+\frac{\beta}{r_{D}} \frac{\partial \eta}{\partial r_{D}}=\frac{r_{D}^{\theta}}{\left(1-\alpha_{D} \eta\right)} \frac{\partial \eta}{\partial t_{D}}$

$\eta\left(r_{D}, 0\right)=0$

$\lim _{r_{D} \rightarrow 0}\left(r_{D}^{\beta} \frac{\partial \eta}{\partial r_{D}}\right)=-1$

$\lim _{r_{D} \rightarrow \infty} \eta=0$

The strong nonlinearities in Eq.(7) are thus considerably weakened in Eq.(12) and are restricted to the coefficient of the nonlinear term on the right side only.

Introduce a parameter perturbation in $\alpha_{D}$ by defining the series

$$
\eta=\eta_{0}+\alpha_{D} \eta_{1}+\alpha_{D}^{2} \eta_{2}+\cdots
$$

and expanding the coefficient of the partial derivative on the right side in a binomial series with the stipulation that $\alpha_{D} \eta<1$, we obtain the zeroth-order approximate equation

$\frac{\partial^{2} \eta_{0}}{\partial r_{D}^{2}}+\frac{\beta}{r_{D}} \frac{\partial \eta_{0}}{\partial r_{D}}=r_{D}^{\theta} \frac{\partial \eta_{0}}{\partial t_{D}}$

The initial and boundary value conditions are also Eqs.(13)-(15).

In order to obtain the zeroth-order approximate solution, the following transforms are introduced:

$$
u=d_{f}^{2-\frac{\theta+2}{d_{f}}} \eta_{0}, x=\frac{1}{d_{f}} r_{D}^{d_{f}}, t_{1}=d_{f}^{2-\frac{\theta+2}{d_{f}}} t_{D}
$$

The zeroth-order approximate initial and boundary value problem become

$$
\frac{\partial}{\partial x}\left(x^{2-\frac{\theta+2}{d_{f}}} \frac{\partial u}{\partial x}\right)=\frac{\partial u}{\partial t_{1}}
$$

$\lim _{x \rightarrow 0} x^{2-\frac{\theta+2}{d_{f}}} \frac{\partial u}{\partial x}=-1 \quad t_{1}>0$

$\lim _{x \rightarrow \infty} u=0 t_{1}>0$

$\left.u\right|_{t_{1}=0}=0 \quad 0 \leq x \leq \infty$

The above zeroth-order approximate initial and boundary value problem can be seen as the continuous source problem. First, we solve the instantaneous source problem corresponding to the continuous source problem.

The instantaneous source problem is formulated with Eqs.(19)-(22)

$$
\frac{\partial}{\partial x}\left(x^{2-\frac{\theta+2}{d_{f}}} \frac{\partial u}{\partial x}\right)=\frac{\partial u}{\partial t_{1}}
$$

$\lim _{x \rightarrow 0} x^{2-\frac{\theta+2}{d_{f}}} \frac{\partial u}{\partial x}=0 \quad t_{1}>0$ 


$$
\lim _{x \rightarrow \infty} u=0
$$

$\left.u\right|_{t_{1}=0}=\delta(x) \quad 0 \leq x \leq \infty$

where $\delta(x)$ is the Dirac- $\delta$ function

Let us seek similarity_solutions of Eq.(19) subject to conditions (20), (21) and (22) by introducing the similarity transforms

$u=w(\rho) t_{1}^{-m}, \quad \rho=x t_{1}^{-m}, \quad m>0$

Substituting transforms (23) into Eq. (19), we find that similarity demands that

$$
m=\frac{d_{f}}{\theta+2}=1-v
$$

For $d_{f}>0, \theta>0$, the requirement $m>0$ is satisfied. Use of transforms (23) and Eq.(24) reduces Eq.(19) to the ordinary differential equation

$$
-\left(\frac{d_{f}}{\theta+2}\right) \frac{\mathrm{d}}{\mathrm{d} \rho}(\rho w)=\frac{\mathrm{d}}{\mathrm{d} \rho}\left(\rho^{2-\frac{\theta+2}{d_{f}}} \frac{\mathrm{d} w}{\mathrm{~d} \rho}\right)
$$

subject to

$$
\lim _{\rho \rightarrow 0} \rho^{2-\frac{\theta+2}{d_{f}}} \frac{\mathrm{d} w}{\mathrm{~d} \rho}=0
$$

Two integrations give

$$
w(\rho)=w_{0} \exp \left[-\left(\frac{d_{f}}{\theta+2}\right)^{2} \rho^{\frac{\theta+2}{d_{f}}}\right]
$$

with $w_{0}$ the value of $w(\rho)$ at $\rho=0$.

We complete the solution by noting that

$$
\int_{0}^{\infty} u(x) \mathrm{d} x=1
$$

so

$$
\int_{0}^{\infty} w(\rho) \mathrm{d} \rho=1
$$

That is,

$$
\begin{gathered}
w_{0}=\left\{\int_{0}^{\infty} \exp \left[-(1-v)^{2} \rho^{\frac{1}{1-v}}\right] \mathrm{d} \rho\right\}^{-1}= \\
{\left[\frac{1}{(1-v)^{1-2 v}} \Gamma(1-v)\right]^{-1}}
\end{gathered}
$$

As Duhamel's principal, we secure the continuous source solutions by integrating the instantaneous source solution with respect to $t$

$$
\begin{gathered}
u=\int_{0}^{1} w_{0} \exp \left[-(1-v)^{2} \rho^{\frac{1}{1-v}}\right] t_{1}^{-(1-v)} \mathrm{d} t_{1}= \\
w_{0} \int_{0}^{1} t_{1}^{-(1-v)} \exp \left[-(1-v)^{2} x^{\frac{1}{1-v}} t_{1}^{-1}\right] \mathrm{d} t_{1}
\end{gathered}
$$

Making transform $y=(1-v)^{2} x^{\frac{1}{1-v}} t_{1}^{-1}$ gives

$$
\begin{gathered}
u=w_{0}\left[(1-v)^{2} x^{\frac{1}{1-v}}\right]^{v} \int_{y}^{\infty} y^{-(1+v)} \exp (-y) \mathrm{d} y= \\
w_{0}\left[(1-v)^{2} x^{\frac{1}{1-\nu}}\right]^{v} \Gamma(-v, y)
\end{gathered}
$$

where $\Gamma(a, y)$ is the incomplete Gamma function Inserting Eqs.(28) and (29) into Eq.(18), we get

$$
\eta_{0}=\frac{r_{D}^{\theta+2-d_{f}}}{(\theta+2) \Gamma(1-v)} \Gamma\left[-v, \frac{r_{D}^{\theta+2}}{(\theta+2)^{2} t_{D}}\right]
$$

(2)The problem of fluid flow in an infinite reservoir with the constant pressure production is as follows:

$\frac{\partial^{2} p_{D}}{\partial r_{D}{ }^{2}}+\frac{\beta}{r_{D}} \frac{\partial p_{D}}{\partial r_{D}}-\alpha_{D}\left(\frac{\partial p_{D}}{\partial r_{D}}\right)^{2}=r_{D}{ }^{\theta} \mathrm{e}^{\alpha_{D} p_{D}} \frac{\partial p_{D}}{\partial t_{D}}$

$p_{D}\left(r_{D}, 0\right)=0$

$\lim _{r_{D} \rightarrow 0} p_{D}=1$

$\lim _{r_{D \rightarrow \infty}} p_{D}=0$

By using transform (11), the above flow problem 
is simplified as

$\frac{\partial^{2} \eta}{\partial r_{D}^{2}}+\frac{\beta}{r_{D}} \frac{\partial \eta}{\partial r_{D}}=\frac{r_{D}^{\theta}}{\left(1-\alpha_{D} \eta\right)} \frac{\partial \eta}{\partial t_{D}}$

$\eta\left(r_{D}, 0\right)=0$

$\lim _{r_{D} \rightarrow 0} \eta=\frac{1-\mathrm{e}^{\alpha_{D}}}{\alpha_{D}}$

$\lim _{r_{D} \rightarrow \infty} \eta=0$

\section{(a) Zeroth order:}

The zeroth-order approximate equation is also Eq.(17), The initial and boundary value conditions are given by Eqs.(32) (33) and (34)

Making use of transformation expressed by Eq.(35)

$$
y=\frac{r_{D}^{\theta+2}}{(\theta+2)^{2} t_{D}}
$$

reduces the initial-boundary value problem of partial differential equation to the boundary value problem of ordinary differential equation as follows:

$\frac{\mathrm{d}^{2} \eta_{0}}{\mathrm{~d} y^{2}}+\left(1+\frac{1-v}{y}\right) \frac{\mathrm{d} \eta_{0}}{\mathrm{~d} y}=0$

$\lim _{y \rightarrow 0} \eta_{0}=1$

$\lim _{y \rightarrow \infty} \eta_{0}=0$

which has the solution

$\eta_{0}=C_{1} \int_{y}^{\infty} y^{-(1-v)} \mathrm{e}^{-y} \mathrm{~d} y+C_{2}$

Using the initial and boundary value conditions, we get

$C_{2}=0, C_{1}=\frac{1}{\Gamma(v)}$

And the solution is given by

$\eta_{0}=\frac{\Gamma(v, y)}{\Gamma(v)}$ (b) First order:

$\frac{\partial^{2} \eta_{1}}{\partial r_{D}^{2}}+\frac{\beta}{r_{D}} \frac{\partial \eta_{1}}{\partial r_{D}}=r_{D}^{\theta}\left(\frac{\partial \eta_{1}}{\partial t_{D}}+\eta_{0} \frac{\partial \eta_{0}}{\partial t_{D}}\right)$

$\eta_{1}\left(r_{D}, 0\right)=0$

$\lim _{r_{D} \rightarrow 0} \eta_{1}=-\frac{1}{2}$

$\lim _{r_{D} \rightarrow \infty} \eta_{1}=0$

By using Eq.(35), the above equation is expressed as

$\frac{\mathrm{d}^{2} \eta_{1}}{\mathrm{~d} y^{2}}+\left(1+\frac{1-v}{y}\right) \frac{\mathrm{d} \eta_{1}}{\mathrm{~d} y}=-\eta_{0} \frac{\mathrm{d} \eta_{0}}{\mathrm{~d} y}$

where

$\eta_{0} \frac{\mathrm{d} \eta_{0}}{\mathrm{~d} y}=-\frac{1}{[\Gamma(v)]^{2}} y^{-(1-v)} \mathrm{e}^{-y} \Gamma(v, y)$

Substituting Eq.(38) into Eq.(37) yields

$$
\begin{aligned}
\frac{\mathrm{d}^{2} \eta_{\mathrm{I}}}{\mathrm{d} y^{2}}+ & \left(1+\frac{1-v}{y}\right) \frac{\mathrm{d} \eta_{1}}{\mathrm{~d} y}= \\
& \frac{1}{[\Gamma(v)]^{2}} y^{-(1-v)} \mathrm{e}^{-y} \Gamma(v, y)
\end{aligned}
$$

Equation (39) can be solved by the method of variation of parameters. The homogeneous solutions are

$u_{1}=-\Gamma(v, y), u_{2}=1$

where $u_{1}$ and $u_{2}$ are intermediate solution values.

Let

$h(y)=\frac{1}{[\Gamma(v)]^{2}} y^{-(l-v)} \mathrm{e}^{-y} \Gamma(v, y)$

The Wronskian of the homogeneous solutions is given by

$W=\left|\begin{array}{ll}u_{1} & u_{2} \\ u_{1}^{\prime} & u_{2}^{\prime}\end{array}\right|=-y^{-(1-v)} \mathrm{e}^{-y}$ 
The general solution to Eq. (37) is given by

$$
\eta_{1}=C_{1}(y) u_{1}(y)+C_{2}(y) u_{2}(y)
$$

where

$$
\begin{gathered}
C_{1}(y)=\int^{y} C_{1}^{\prime}(y) \mathrm{d} y=\int^{y}-\frac{h u_{2}}{W} \mathrm{~d} y=\frac{1}{[\Gamma(v)]^{2}} \cdot \\
{[y \Gamma(v, y)-\Gamma(1+v, y)]+C_{3}}
\end{gathered}
$$

and

$$
\begin{aligned}
C_{2}(y)= & \int C_{2}^{\prime}(y) \mathrm{d} y=\int \frac{h u_{1}}{W} \mathrm{~d} y=\frac{1}{[\Gamma(v)]^{2}} \cdot \\
& \left\{(y-v)[\Gamma(v, y)]^{2}-2 y^{v} \mathrm{e}^{-y} \Gamma(v, y)+\right. \\
& \left.2^{1-2 v} \Gamma(2 v, 2 y)\right\}+C_{4}
\end{aligned}
$$

Substituting Eqs.(41) and (42) into Eq.(40) and using boundary conditions lead to

$$
C_{4}=0, C_{3}=\frac{2^{1-2 v} \Gamma(2 v)}{[\Gamma(v)]^{3}}+\frac{1}{2 \Gamma(v)}
$$

Thus, on simplification, the first-order solution becomes

$$
\begin{gathered}
\eta_{1}=\frac{1}{[\Gamma(v)]^{2}}\left[2^{1-2 v} \Gamma(2 v, 2 y)-y^{v} \mathrm{e}^{-y} \Gamma(v, y)-\right. \\
\left.\frac{2^{2-2 v} \Gamma(2 v)+[\Gamma(v)]^{2}}{2 \Gamma(v)} \Gamma(v, y)\right]
\end{gathered}
$$

\section{Discussion}

The transformation given by Eq.(11) can be rewritten as

$$
p_{D}=-\frac{1}{\alpha_{D}} \ln \left[1-\alpha_{D} \eta_{0}-\alpha_{D}^{2} \eta_{1}+O\left(\alpha_{D}^{3}\right)\right]
$$

Figure 1 demonstrates the variation of dimensionless pressure with time for different values of $\alpha_{D}$, namely, $0.01,0.1,0.2$ and 0.5 . It can be seen that the pressure curves are not closely related to the magnitudes of $\alpha_{D}$ in the initial stage. With time increasing, the effect of $\alpha_{D}$ become larger and larger. With the lincrease of $\alpha_{D}$, the dimensionless pressure decreases. Figure 2 shows the temporal pressure changes for the various value of $d_{s}$. A general trend is that pressure is increased faster for the smaller value of $d_{s}$. The effect of $d_{s}$ is smaller in the initial stage, In the transitional stage, the pressure curves start diverging from each other depending on the relative magnitude of $d_{s}$, and the effect of $d_{s}$ becomes the largest. In later stage, the effect of $d_{s}$ becomes smaller and smaller.

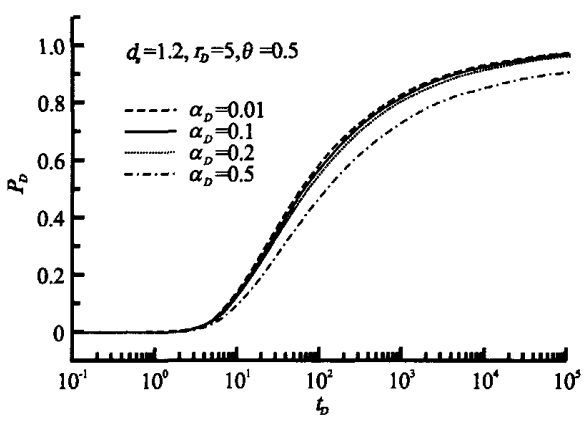

Fig.1 Semi-logarithm plots of pressure versus time depending on $\alpha_{D}$

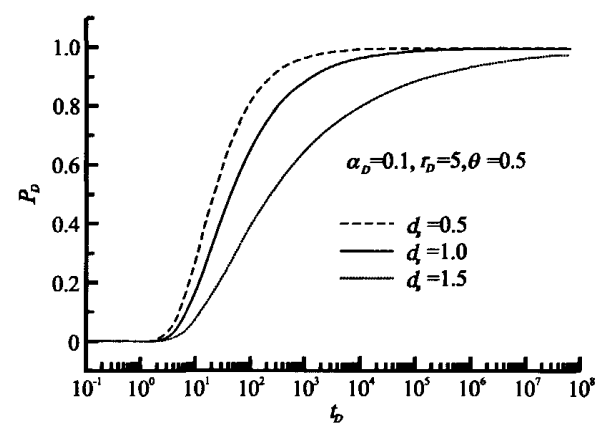

Fig.2 Semi-logarithm plots of pressure versus time at different $d_{s}$

Figure 3 exhibits the temporal variation of the pressure at different radii close to the well bore for two different values of $\alpha_{D}$. In the initial stage, the variation of the pressure is smaller at different radii closer to the well bore. With time increasing, the differences between the pressure curves of fractal reservoir and the pressure curves of stress-sensitive fractal reservoir oncreases. Stress sensitivity in a variety of reservoir situations could be important and needs to be taken into account. Figure 4 shows that magnitude of the pressure curves at any radial 
distance would depend not only on the values of $\alpha_{D}$, but also on time $t_{D}$. For smaller $t_{D}$ (e.g., at $t_{D}=10^{2}$ ), the difference between the pressure curves for the values $\alpha_{D}$ is smaller. For larger $t_{D}$ (e.g., at $t_{D}=10^{4}$ ), the difference between the pressure curves for the values $\alpha_{D}$ is larger. At any time, the difference between pressure curves would increase with increasing distance from the well bore. At large distances, however, pressure curves tend to uniformity., and the effect of the deformed parameter $\alpha_{D}$ died out.

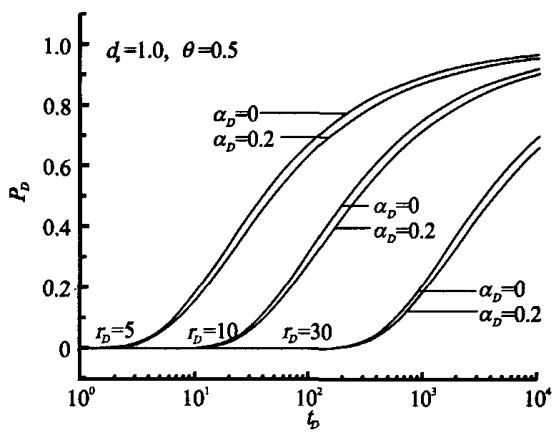

Fig.3 Semilogarithm plots of pressure versus time at different radii

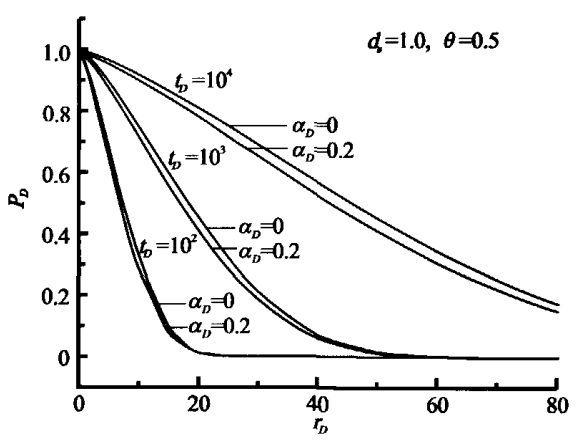

Fig.4 Semilogarithm plots of pressure versus radius at different times

\section{Conclusion}

(1) Fractal dimensions have been introduced to the flow equation of fluids in fractal reservoirs. The flow model has more extensive adaptability compared with the traditional ones.

(2)The similarity solutions for the flow models of fluid in fractal reservoir with both the constant rate production and the constant pressure production have been obtained by using similarity solution method.

(3) The perturbation solutions for a constant pressure production and a constant rate production of linear-source well have been obtained by using the regular perturbation method in an infinitely large system.

(4) With time increasing, the effect of $\alpha_{D}$ becomes remarkable. With increasing $\alpha_{D}$, dimensionless pressure decreases.

\section{References}

[1] PEDROSA O. A. Jr., Pressure transient response in stress-senisitive formation[C]. SPE 15115. California, Oakland, USA, 1986.

[2] TONG Deng-ke, Ge Jia-li. An exact solution for unsteady seepage flow through fractal reservoir[J]. Acta Mechanica Sinica, 1998, 30(5): 621-626 (in Chinese).

[3] TONG Deng-ke, Wang Rui-he. Exact solution and dynamical characteristics of non-Newtonian power-law fluid flow in fractal reservoir[J]. Journal of Hydrodynamics, Ser. A, 2000, 15(3): 342-350(in Chinese).

[4] TONG Deng-ke, Chen Qin-lei. An exact solution and pressure characteristics of the flow of fluid through fractal reservoir[J]. Journal of Hydrodynamics, Ser. A, 1999,14(2): 201-209 (in Chinese).

[5] TONG Deng-ke, WANG Rui-he. The line-source solution and flow analysis of fluid in fractal reservoir[J]. Journal of Hydrodynamics Ser. B, 2002,14(4): 59-65.

[6] TIAN Ji, TONG Deng-ke. The Flow analysis of fluids in fractal reservoir with the fractional derivative[J] Journal of Hydrodynamics, Ser. B, 2006,18(3): 287-293

[7] KIKANI J., PDROSA O. A. Jr. Perturbation analysis of stress-sensitive reservoirs[J]. SPE Form. Eval.,1991, 6(3): 379-386.

[8] YEUNG K., CHAKRABARTY C. An approximate analytical study of aquifers with pressure-sensitive formation permeability[J]. Water Resources Research, 1993, 29(10): 3495-3510.

[9] YANG Yong-zhi, LIAO Xin-wei and SHEN Ping-ping. Evaluation on inflow performance relationship of gas wells in pressure-sensitive reservoir using generalized pseudo pressure $[\mathrm{J}]$. Oil Driling and Production Technology, 2007, 29(1): 26-31(in Chinese).

[10] ZHAO Dong-mei, YAO Jun. The pressure-transient characteristics of a stress-sensitive triple-medium reservoir[J]. Chinese Journal of Computation Physics, 2005, 22(5): 444-448 (in Chinese).

[11] WANG Zi-sheng, YAO Jun. Study of pressure-transient characteristic for stress-sensitive triple-medium reservoirs with fractures and vugs conveying fluids to well bore[J]. Journal of Hydrodynamics, Ser. A, 2006, 21(1): $84-89$ (in Chinese)

[12] TONG Deng-ke, JIANG Dong-mei and CHEN Qin-lei. Dynamic characteristics of reservoir with deformed double-porosity medium $[\mathrm{J}]$. Journal of the University of Petroleum, 2001, 25(5): 53-56(in Chinese).

[13] TIAN Ji, TONG Deng-ke. The nonlinear flow simulation using double permeability model in deformed media [J]. Engineering Mechanics, 2006, 23(7): 40-44 (in Chinese).

[14] MA Xiao-dan,TONG Deng-ke. Non-Darcy flow analysis of fluid in deformed fractal reservoir with double 
porosity[J]. Chinese Journal of Computation Physics, 2007, 24(2): 197-202 (in Chinese).

[15] SHI Li-na, TONG Deng-ke. Pressure analysis of double permeability model in deformed medium with well bore storage effect $[\mathrm{J}]$. Chinese Quarterly of Mechanics, 2006, 27(2): 206-211(in Chinese).
[16] WANG Wen-huan. Three-zone composite well test model of condensate gas reservoir in stress-sensitive sandstone[J]. Petroleum Exploration and Development, 2005, 32(3): 117-119 (in Chinese). 